\title{
No todo es "cut-out": reclasificación de las complicaciones mecánicas del tornillo cefálico del clavo intramedular.
}

\author{
DOI: http://dx.doi.org/10.37315/SOTOCAV201928054136 \\ WADHWANI J, GIL MONZÓ ER, PÉREZ CORREA JI, GARCíA ÁLVAREZ J, BLAS DOBÓN JA, RODRIGO PÉREZ JL. \\ SERVICIO DE CIRUGÍA ORTOPÉDICA Y TRAUMATOLOGÍA, HOSPITAL UNIVERSITARIO DOCTOR PESET, VALENCIA
}

\begin{abstract}
Resumen.
El uso del clavo intramedular en fracturas extracapsulares del tercio proximal de fémur es el sistema más frecuente de estabilización. En los últimos años, las complicaciones biomecánicas que afectan este sistema han ido aumentando.

Se realiza un estudio retrospectivo radiográfico de fracturas intertrocantéricas seguido de un análisis de las complicaciones biomecánicas en la zona 1 del tornillo cefálico. De los 548 casos, se observaron 85 casos (15.5\%) de complicaciones, siendo "back-out" la complicación menor más frecuente y "cut-out" la complicación mayor más frecuente. El $41 \%$ del total de las fracturas corresponden al tipo 31-A2.3 AO/OTA. Las fracturas basicervicales y 31-A2.3 tienen un alto riesgo de complicaciones.

El origen biomecánico que describe Lenich es importante conocerlo debido a que la solución quirúrgica varía según si es cut-out, cut-in (mismo origen del fallo biomecánico del tornillo cefálico) o cut-through (diferente origen) para, en consecuencia, resolverlas de la manera adecuada.
\end{abstract}

Palabras clave: fracturas basicervicales, pertrocantereas, cut-out, cut-in, back-out

\section{Summary.}

The use of the intramedullary nail in extracapsular fractures of the proximal third of the femur is the most frequent stabilization system. In recent years, the biomechanical complications that affect this system have been increasing.

A retrospective radiographic study of intertrochanteric fractures was performed followed by an analysis of the biomechanical complications in zone 1 of the cephalic screw. Of the 548 cases, 85 cases (15.5\%) of complications were observed, with "back-out" being the most frequent minor complication and "cut-out" the most frequent major complication. $41 \%$ of the total fractures correspond to type 31-A2.3 AO / OTA. The basicervical fractures and 31-A2.3 have a high risk of complications.

The biomechanical origin that Lenich describes is important to understand as the surgical solution varies depending on whether it is cut-out, cut-in (same origin of the biomechanical failure of the head screw) or cut-through (different origin) to, consequently, resolve them in the right manner.

\section{Correspondencia:}

Jayant Wadhwani

Hospital Universitario Doctor Peset Valencia

Servicio de Cirugía Ortopédica y Traumatología

Avenida Gaspar Aguilar, 90

46007, Valencia

\section{Introducción}

El uso del clavo intramedular es el sistema más frecuente de estabilización de fracturas extracapsulares del tercio proximal de fémur. En los últimos años, las complicaciones biomecánicas que afectan a este sistema han ido aumentando.

Las complicaciones tras realizar el enclavado intramedular en las fracturas extracapsulares del tercio proximal del fémur pueden dividirse en mecánicas, biológicas y en algún caso, error técnico en la ejecución del enclavado. 
WADHWANI J Y COLS. No todo es "cut-out": reclasificación de las complicaciones mecánicas del tornillo cefálico del clavo intramedular.

Las complicaciones mecánicas dependen de la localización del fallo del implante, encargado de la estabilidad. Se pueden clasificar en tres zonas: zona 1 (relacionado al tornillo cefálico y cabeza femoral), zona 2 (relacionado al clavo intramedular y la zona diafisaria del fémur) y zona 3 (relacionado con el tornillo distal y la punta del clavo intramedular) (Fig. 1).

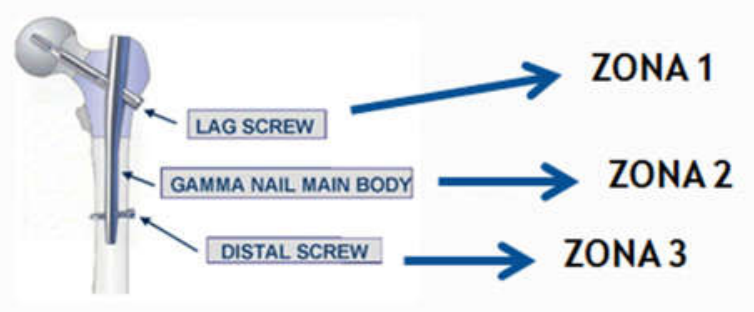

Figura 1: clasificación de las zonas de las complicaciones mecánicas del clavo IM.

Las complicaciones relacionadas con la zona 1 son las más comunes y de mayor enfoque. Se pueden dividir en mayores y menores, según la gravedad. Dentro de las mayores se encuentran el cut-out, cut-in, cut-through, pull-out y migración medial. Entre las complicaciones menores se encuentra el back-out. Es importante un correcto conocimiento de estos términos, por su confusión o interpretación errónea.

Así mismo, las complicaciones se pueden clasificar según la evolución temporal desde el postoperatorio; las complicaciones que ocurren antes de los 2-3 meses se consideran primarias, y más de 3 meses se consideran secundarias relacionadas a una pseudoartrosis, condrolisis, necrosis avascular de cabeza femoral, que están en relación con las complicaciones biológicas (comportamiento de las partes blandas alrededor de la fractura y la calidad ósea).

Se debe tener en consideración complicaciones relacionadas con las técnicas que pueden ocurrir intraoperatoriamente. La correcta reducción en todos los planos de la fractura antes de la introducción del clavo es fundamental, así mismo, el tamaño y la posición de la colocación del tornillo cefálico.

Por ello, el objetivo del estudio es definir y reclasificar radiográficamente en el espectro temporal las complicaciones mecánicas del tornillo cefálico en las fracturas basicervicales, pertrocantéricas y persubtrocantéricas en los pacientes tratados con clavo IM (Gamma3 @).

\section{Material y Métodos}

Se realizó un estudio retrospectivo de pacientes adultos mayores de 65 años intervenidos de fracturas de extremo proximal de fémur, según la clasificación de
AO: fracturas pertrocantéricas y persubtrocantéricas (AO/OTA31-A), y basicervicales (AO/OTA 31-B3), tratados con clavos intramedulares tipo Gamma3 $®$.

Se recogen datos de 880 pacientes intervenidos entre enero 2013 a enero 2018 de nuestro Centro. A continuación, se aplicaron unos criterios de exclusión: posteriores

Pacientes sin controles en los seguimientos

- Pacientes operados con otro tipo de osteosíntesis, descartando PFNA o DHS.

- Fracturas patológicas de causa oncológica / fracturas abiertas etc.

- Fracturas diafisarias tratadas con Gamma3®

largo

Se obtuvieron 548 casos, en los cuales se realizaró un análisis radiológico preoperatorio para clasificar las fracturas según la clasificación AO/OTA, junto con las radiografías seriadas a las 4-6 semanas y a los 3, 6 y 12 meses tras la intervención quirúrgica para cada paciente, evaluándose las complicaciones biomecánicas que ocurriron en el área del tornillo cefálico y el estadiaje de su aparición.

Las complicaciones mecánicas que afectan al tornillo cefálico se definen de la siguiente forma (Fig. 2):

- $\quad$ El "cut-out" es una complicación mayor en la cual existe una perforación cefálica con rotación y colapso en varo por migración antero-superior del tornillo.

- $\quad$ El "cut-in" también existe una perforación de la cabeza femoral con rotación y colapso en varo pero existe una migración central del tornillo.

En el "cut-through" existe una perforación central en la articulación coxofemoral sin telescopaje ni aflojamiento del tornillo cefálico.

En el "pull-out" existe una salida descontrolada del tornillo cefálico a través del orificio del clavo por fallo o ausencia del set screw.

- La "migración medial" se define como la progresión del "cut-in" dentro de la pelvis por fallo del set screw.

- $\quad$ El "back-out" es la complicación menor en la cual existe una migración lateral $(>1 \mathrm{~cm})$ del tornillo cefálico sin pérdida del anclaje cefálico habitualmente por colapso de la fractura. 
WADHWANI J Y COLS. No todo es "cut-out": reclasificación de las complicaciones mecánicas del tornillo cefálico del clavo intramedular.
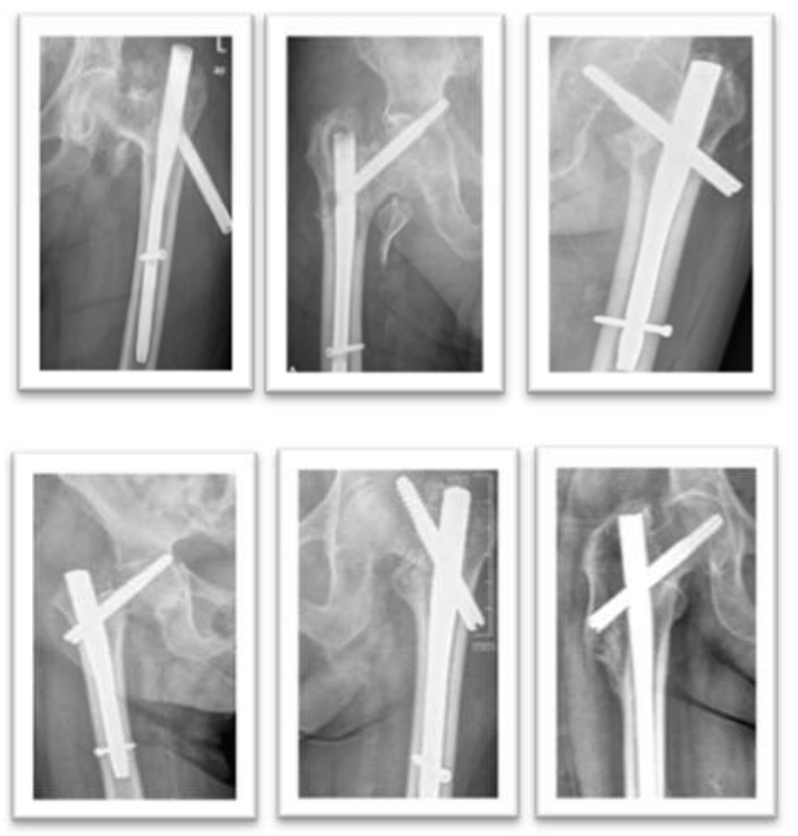

Figura 2 (izquierda - derecha): pull-out, migración medial, back-out, cut-in, cut-out, cut-through

Algunas de estas complicaciones tienen un origen común pudiéndose clasificar dentro de los estadíos de Lenich (Fig. 3), basada en las fases de cambios mecánicos del tornillo cefálico: Lenich fase 1: rotación de la cabeza femoral (momentos torsionales), Lenich fase 2: migración desanclaje del tornillo haciendo un "efecto limpiaparabrisas", Lenich fase 3: colapso en varo y rasgado de cabeza en dirección cefálica/axial. En nuestro estudio radiográfico, re-clasificaremos las complicaciones con este origen.

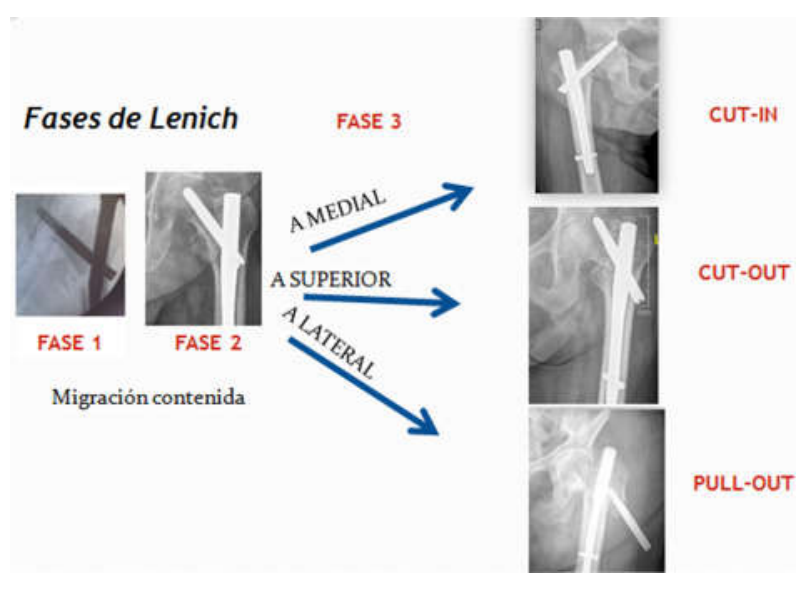

Figura 3: Complicaciones mecánicas según las fases de Lenich.

\section{Resultados}

Se recogieron un total de 880 pacientes con estos tipos de fracturas que fueron tratados con clavo Gamma $3 \AA$. De esta muestra, 548 casos cumplieron los criterios de inclusión.
La edad media de los pacientes incluidos fue de 84.3 años (65-103) (Fig. 4) de los cuales 126 (23.0\%) eran hombres y $422(77.0 \%)$ mujeres de la población

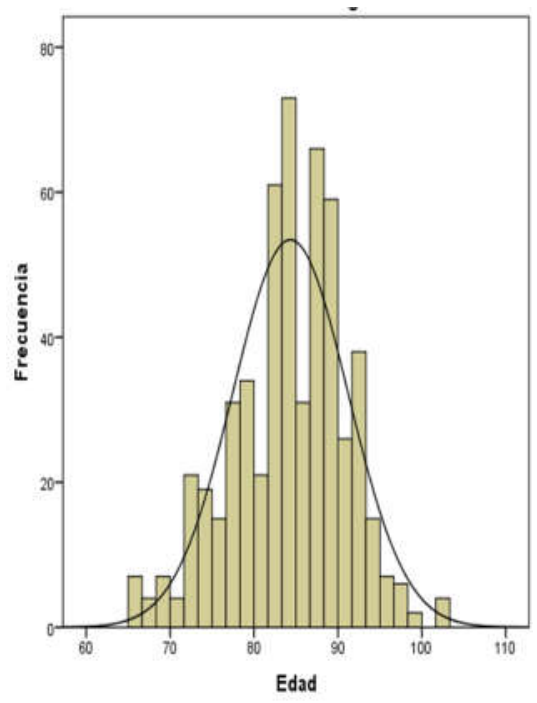

estudiada. De los 548 pacientes, 135 pacientes (25\%) fueron tratados con Gamma $3 \AA$ largo y el 413 pacientes $(75 \%)$ con Gamma $3 \AA$ corto. Se analizaron todas las radiografías de la muestra y se clasificaron según el tipo de fractura de la clasificación de la $A O$, así mismo dividiéndolas por subtipos.

Se presentaron 64 casos (11.7\%) de 31-A1.2, 25 casos (4.6\%) de 31-A2.2, 60 casos (10.9\%) de 31-A3.3, 154 pacientes $(28.1 \%)$ con $31-\mathrm{A} 1.3,225$ pacientes $(41 \%)$ de 31-A2.3, esta última siendo la más frecuente de los subtipos. Se obtuvieron solo 20 casos (3.6\%) de fracturas basicervicales o de subtipo 31-B3 (Fig. 5).

Con respecto al tipo de complicaciones, se obtuvieron un total de 85 casos (15.5\%) del total de casos que sufrieron complicaciones, de las cuales 26 (30.6\%) pacientes eran mayores y 59 (69.4\%) eran menores (Tabla I).

Al analizar los datos con más detenimiento con respecto a las complicaciones biomecánicas que se producía según el tipo de fractura (Fig. 6), de los 59 casos de back-out, 31 de ellos (53\%) correspondían a fracturas 31-A2.3, siendo esta complicación la más frecuente. El back-out representa el $10.6 \%$ de todos los 548 casos, siendo la complicación más frecuente, especialmente en las fracturas 31-A2.3 y 31-B3 detectándose en 59 casos (aproximadamente $70 \%$ de las complicaciones de todas las complicaciones del tornillo cefálico). El 53\% de las fracturas 31-A2.3 sufrieron colapso y telescopaje del tornillo cefálico de aproximadamente $1 \mathrm{~cm}$.

Al dividir el tipo de fractura con respecto al número total de casos, resulta que existe más riesgo de back-out en las fracturas con más conminución en el foco, (32\% en 31-A2.2 y 13.8\% en 31-A2.3). (Tabla II y Fig. 7). 
WADHWANI J Y COLS. No todo es "cut-out": reclasificación de las complicaciones mecánicas del tornillo cefálico del clavo intramedular.

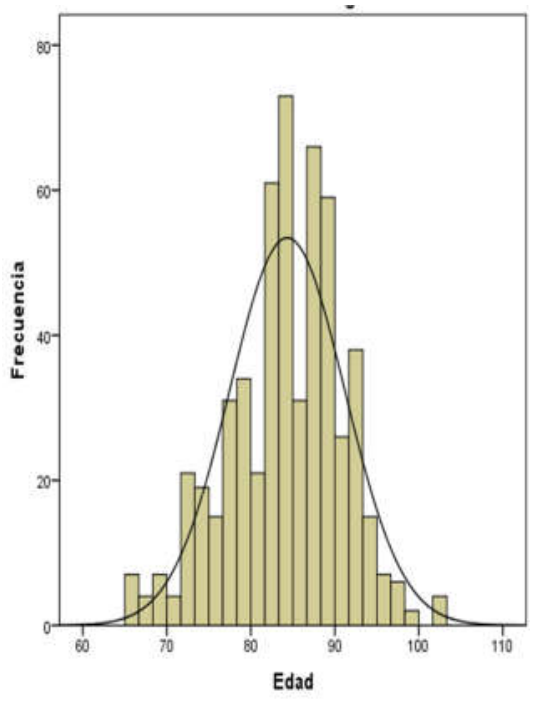

Figura 4: histograma de barras de la media de edad de pacientes que sufrieron fracturas intertrocantéricas.

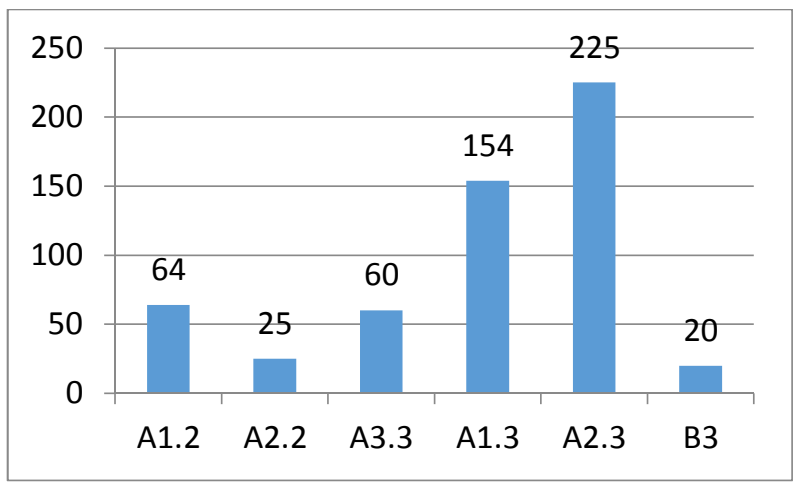

Figura 5: histograma de barras de número total de casos, según el tipo de fractura

\begin{tabular}{|ll|ll|}
\hline \multicolumn{2}{|c|}{ Complicaciones } & Mayores: 26 & \multicolumn{2}{|c|}{ Complicaciones menores: 59} \\
\hline Cut-Out: 18 & $(21.2 \%)$ & Back-Out: 59 & $(69.4 \%)$ \\
\hline Cut-Through: 6 & $(7 \%)$ & & \\
\hline Cut-In: 2 & $(2.4 \%)$ & & \\
\hline
\end{tabular}

Tabla I: Complicaciones mecánicas divididas en mayores y menores.

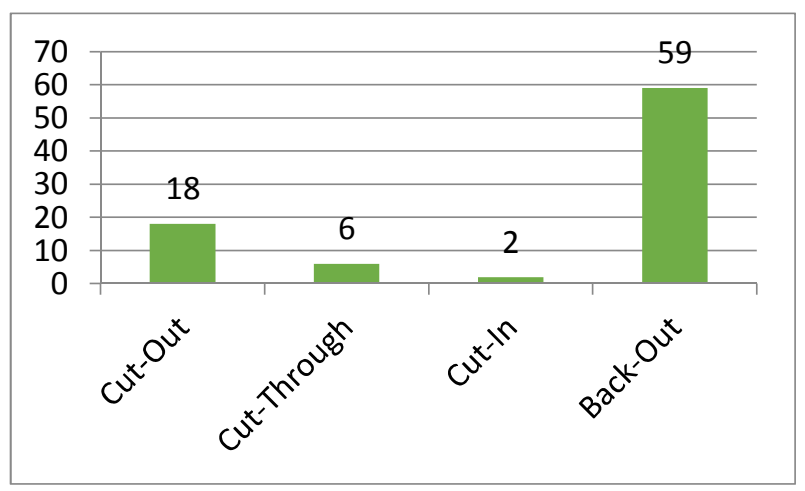

Figura 6: número total de casos de cada complicación biomecánica

Dentro de las complicaciones mayores (cut-out, pull-out, cut-in, cut-through) se detectaron 18 casos de cut-out (de los cuales 10 pacientes se detectaron en Lenich fase 2 y 8 casos en Lenich fase 3 ), siendo más frecuente en las fracturas $31-\mathrm{B} 3$ con 7 casos siendo esto el $35 \%$ del total de estas todas las fracturas (20 pacientes) y en las fracturas $31-\mathrm{A} 2.3$ con 5 casos siendo el $2.2 \%$ del total de estas todas las fracturas (225 pacientes).

Como segunda complicación en frecuencia se observó el cut-through en 6 casos y también fue más frecuente en las fracturas tipo $31-\mathrm{A} 2.3$ con 4 casos, que supone el $1.7 \%$. Solo hubo 2 casos de cut-in, 1 en las fracturas $31-B 3$ y otra en la fractura $31-A 2.3$. El $39 \%$ de los 18 casos se obtuvieron en fracturas basicervicales o 31-B3. (Tabla III).

\begin{tabular}{|l|c|c|c|c|c|c|}
\hline & \multicolumn{3}{|c|}{ COLAPSO } & TOTAL & $\begin{array}{c}\text { total / } \\
\text { casos }\end{array}$ & $\begin{array}{c}\text { total / tipo } \\
\text { fractura }\end{array}$ \\
\hline & $1 \mathrm{~cm}$ & $<1 \mathrm{~cm}$ & $>\mathbf{1} \mathrm{cm}$ & & $\%$ & $\%$ \\
\hline $31 \mathrm{~A} 2.3$ & 8 & 11 & 12 & $31(53 \%)$ & 5.6 & 13.8 \\
\hline $31 \mathrm{~A} 3.3$ & 3 & 1 & 2 & 6 & 1.1 & 10 \\
\hline $31 \mathrm{~A} 1.3$ & 1 & 6 & 3 & 10 & 1.8 & 6.5 \\
\hline $31 \mathrm{~A} 1.2$ & & 2 & 2 & 4 & 0.7 & 6.3 \\
\hline $31 \mathrm{A2} 2$ & & 4 & 4 & 8 & 1.4 & 32 \\
\hline TOTAL & 11 & 23 & 23 & 59 & 10.6 & \\
\hline
\end{tabular}

Tabla II: Resultados de back-out con un total de 59 casos $(10.6 \%$ del total de casos), siendo las más frecuentes de tipo $31 \mathrm{~A} 2.2$ y $31 \mathrm{~A} 2.3$.

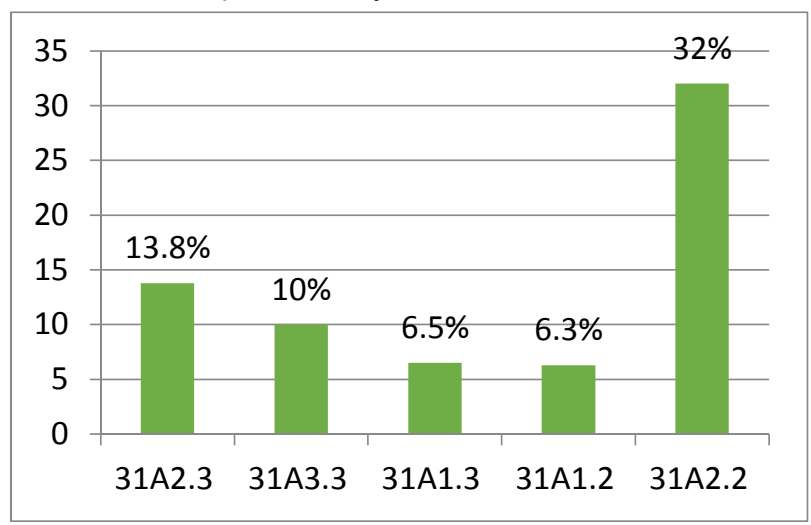

Figura 7: Porcentajes del total número de casos de "back-out" con respecto a cada tipo de fractura. 
WADHWANI J Y COLS. No todo es "cut-out": reclasificación de las complicaciones mecánicas del tornillo cefálico del clavo intramedular.

\begin{tabular}{|c|c|c|c|c|} 
& CUT-THROUGH & CUT-OUT & CUT-IN & TOTAL \\
\hline 31 A2.3 & 4 & $5(28 \%)$ & 1 & 10 \\
\hline 31 A1.3 & 0 & 3 & 0 & 3 \\
\hline 31 A1.2 & 1 & 3 & 0 & 4 \\
\hline $31 \mathrm{~A} 2.2$ & 1 & 0 & 0 & 1 \\
\hline 31 B3 & 0 & $7(39 \%)$ & 1 & 8 \\
\hline TOTAL & 6 & $18^{*}$ & 2 & 26 \\
\hline Total/casos & $1.09 \%$ & $3.28 \%$ & $0.36 \%$ & \\
\hline
\end{tabular}

Tabla III: Resultados de las complicaciones mecánicas mayores según el tipo de fractura, siendo la más frecuente la fractura $31 \mathrm{~A} 2.3$ y la complicación más frecuente, el cut-out.

\section{Discusión}

Las fracturas intertrocantéricas son una de las fracturas más frecuentes en la población mayor. Sin embargo, el manejo de este tipo de fracturas es un desafío debido al hecho de que es mucho más complejo. El tratamiento de estas fracturas está sujeto a controversia, particularmente sobre el manejo adecuado para evitar complicaciones posteriores. Para facilitar el proceso de toma de decisiones, es importante comprender y evaluar esta complejidad al realizar maniobras de reducción y antes de la cirugía.

Analizando los resultados obtenidos de este estudio, el tipo de fractura intertrocantérica más predominante en nuestro estudio es la 31-A2.3, que presenta unas características distintas con respecto al resto de trazos. Presenta una gran conminución en el foco de fractura. Esta conmunición dificulta delimitar el trazo de fractura en la escopia, así mismo dificulta la correcta alineación de la fractura. Ese desplazamiento de esta fractura es difícil de predecir por su inestabilidad, conllevando el fragmento proximal o cabeza del fémur que se desplace en valgo o varo.

Barra et al. ${ }^{1}$, también verifican que las fracturas más frecuentes corresponden al grupo $31-\mathrm{A} 2$ hasta un $57 \%$ de los casos.

En el estudio de Bojan et al. ${ }^{2}$, los resultados de complicaciones mayores tipo cut-out, corresponde a un $1,85 \%$ de causa biomecánica. Con respecto a nuestro estudio, el cut-out corresponde a un $3.28 \%$ de todos los casos.

Cabe destacar que a pesar de una reducción correcta y fijación interna mediante clavo endomedular, este tipo de fractura (AO 31-A2) es la que más complicaciones ha generado en este estudio: $38 \%$ de las complicaciones mayores y $59 \%$ de las complicaciones menores.

Estas complicaciones se pueden explicar mediante un cambio biomecánico en el foco de fractura cuando el paciente comienza la carga y la marcha sobre el miembro intervenido. El colapso del cuello femoral y las fuerzas de cizallamiento en una zona conminuta, alrededor de tornillo cefálico pueden producir un colapso en el área de la fractura.

En un estudio realizado por Xiaowei Huang et al ${ }^{3}$, concluyen que en fracturas 31-A3, la osteosíntesis tipo DHS no es adecuado por la falta de integridad de la región del calcar, y mostró un mayor desplazamiento del fragmento fracturado. Generalmente, el clavo Gamma tiene una superioridad mecánica sobre el DHS. Cuando la integridad de la región calcar no se mantiene, se prefiere osteosíntesis con un clavo intramedular.

Con respeto a las fracturas basicervicales o 31-B3, tuvimos 20 casos en el estudio de las cuales no se detectaron casos de complicaciones menores y 7 casos de cut-out. Esto corresponde al $35 \%$ casos en casos tratados con fijación mediante clavo endomedular. Aunque hayan pocos casos de este tipo de fractura, el riesgo de complicación es muy alto, es decir, 1 caso de cada 3 pacientes presentan un riesgo de cut-out. $72 \%$ de los casos evaluados correspondían a fracturas basicervicales o comnimutas ${ }^{2}$.

En estudios previamente publicados, existen otros factores de riesgo para que se produzcan estas complicaciones biomécanicas: osteoporosis 4 , colocación del tornillo cefálico en el cuadrante de Cleveland ${ }^{5}$, la distancia tip-apex (TAD o tip-apex distancia) según Baumgartner ${ }^{6}$, la angulación y longitud del tornillo cefálico, el tipo de tornillo cefálico utilizado etc.

El auge de las complicaciones del tornillo cefálico ha enfocado la investigación en el comportamiento del sistema. Lenich et al 7 , concluye que estas complicaciones siguen un estadiaje que pueden ser responsables del movimiento de la cabeza femoral y/o tornillo cefálico. Lenich describe la existencia de tres fases mediante estudios radiográficos:

En la Fase 1 existen momentos torsionales y el tornillo se desplaza de distal a proximal en la cabeza femoral sin presencia de osteólisis alrededor del tornillo, que se aprecia como una varización cefálica que se relaciona con la rotación de la cabeza femoral.

En la Fase 2 existe una migración del implante y de la cabeza femoral debido a una desanclaje del tornillo a efecto "limpiaparabrisas", pudiéndose observar un ascenso progresivo del tornillo. Se produce una microrrotura de las trabéculas alrededor del tornillo, el cual es responsable del anclaje y estabilidad del sistema. Radiológicamente, se aprecia como una osteólisis de cabeza femoral. 
WADHWANI J Y COLS. No todo es "cut-out": reclasificación de las complicaciones mecánicas del tornillo cefálico del clavo intramedular.

En la Fase 3, el comportamiento del tornillo es singular: las migraciones inestables no contenidas dependen si la dirección de la migración es medial (cut-in), hacia arriba (cut-out) o lateral (pull-out). Cut-in tiene la particularidad de que existe un "efecto de succión" del tornillo cefálico hacia el interior, en relación con el resto del tornillo. Esto ocurre por un colapso de la cabeza en varo y rasgado en la cabeza femoral.

Cabe destacar que las primeras dos fases son comunes para las complicaciones que aparecen en la fase 3 y existe una migración contenida.

Las limitaciones del estudio incluyen: la calidad disminuida de las radiografías dificultando la clasificación de las fracturas; la importancia de que la clasificación hecha por un solo cirujano eliminando el sesgo interobservador, dificultad de detección complicaciones en Lenich fase 1 por su aparición precoz.

En las etapas más precoces, la detección y actuación debe ser de manera agresiva, para valorar un tratamiento definitivo evitando así la migración del tornillo cefálico, que puede producir una mayor destrucción de la articulación coxo-femoral. Por ello, un diagnóstico correcto de las complicaciones, ayuda orientar el enfoque terapéutico, siendo más conservador en casos de complicaciones menores y más agresivo en complicaciones mayores.

\section{Conclusiones}

El origen del fallo mecánico en la migración inestable del tornillo cefálico se debe a la existencia de microfracturas trabeculares alrededor del mismo, en primer lugar, por un movimiento sumatorio rotacional de las fuerzas sobre el implante en la cabeza femoral cuando el paciente comienza a realizar carga completa, lo que provoca su aflojamiento progresivo. Este origen biomecánico que describe Lenich es importante conocerlo debido a que la solución quirúrgica de estas complicaciones varía según si es cut-out, cut in (mismo origen del fallo biomecánico del tornillo cefálico) o cutthrough (diferente origen) para en consecuencia resolverlas de la forma adecuada.

El cut-out es la complicación mayor más frecuente, y se da en mayor medida en las fracturas basicervicales 31B3 y pertrocantéreas 31-A2.3 soliendo ocurrir en las primeras seis semanas tal y como hemos detectado en las radiografías seriadas. Aun así, no se debería descartar otras complicaciones menos comunes pero también importantes como el cut-through, cut-in, backout...

Las complicaciones se re-clasificaron según gravedad (mayores y menores) y los estadios de Lenich porque de ello dependen de la repercusión y el tratamiento futuro para su resolución (extracción de material, cambio de tornillo cefálico o cementación del mismo, próstesis total o parcial).

Es por ello que se recomienda realizar una radiografía de control antes de finalizar el primer mes del postoperatorio para una detección precoz de estas complicaciones y que el fallo biomecánico no progrese. De esta forma, se podría detectar precozmente y resolver de forma más sencilla y eficaz teniendo en cuenta el tipo de paciente que suele presentar alta complejidad médica. 
WADHWANI J Y COLS. No todo es "cut-out": reclasificación de las complicaciones mecánicas del tornillo cefálico del clavo intramedular.

\section{Bibliografía}

1. Barra A, Fernández-Gallart J, Jolín T, Gomar F.; The value of crew placement and the apex distance in pertrocanteric fractures treated with gamma nail 2002; 37: 209.

2. Bojan AJ, Beimel C, Taglang G, Collin D, Ekholm C, Jönsson A. Critical factors in cut-out complication after Gamma Nail treatment of proximal femoral fractures. BMC Musculoskelet Disord 2013; 14:1.

3. Huang X, Yu B, Gu Y, Li Z. Biomechanical comparison of dynamic hip screw and Gamma nail for the treatment of unstable trochanteric fractures: a finite element study. Int J Clin Exp Med 2017; 10(5):7867-74.

4. Singh $M$, Nagrath $A$, Maini $P$. Changes in trabecular pattern of the upper end of the femur as an index of osteoporosis. J Bone Joint Surg 1970; 52A:457-67.

5. Cleveland M, Bosworth D, Thompson F, Wilson H, Ishizuka T. A 10 year analisis of intertrochanteric fractures of the femur. J Bone Joint Surg 1959; 41 A:1399-408.

6. Baumgaertner M, Curtin S, Lindskog D, Keggi J. The value of the tip-apex distance in predicting failure of fixation of peritrochaneric hip fractures. J Bone Joint Surg 1995; 77A:1058-64.

7. Lenich A, Bachmeier S, Prantl L. Is the rotation of the femural head a potential initiation for cutting out? A theoretical and experimental approach. BMC Musculoskelet Disord 2011; 12:79. 